\title{
Estudio para la creación de un restaurante polifuncional del sector turístico como alternativa dinamizadora de la economía en cantón Pedernales.
}

\author{
Study for the creation of a multifunctional restaurant in the tourism sector \\ as a dynamic alternative for the economy in the Pedernales canton.
}

Ing. Vladimir Álvarez Ojeda. ${ }^{1}$ \& Ing. Evelyn Fernanda Alcívar Barreno. ${ }^{2}$

\section{Resumen.}

Se realiza un estudio sobre la polifuncionalidad de los locales turísticos recreativos en el cantón Pedernales, como ofertas que integran múltiples servicios en un mismo espacio. En una primera etapa se hace un estudio de mercado que facilita la determinación de la demanda y la perspectiva de la oferta turística para el proyecto, sobre la base de la aplicación de instrumentos de recopilación de información aplicados a clientes. El análisis organizacional y administrativo, así como la estructura interna del local polifuncional se detallan previos a la definición de los aspectos económicos. Con el estudio financiero se determinó el monto de la inversión adecuada para la implementación del estudio, así como los indicadores financieros que muestran la factibilidad económica del proyecto y sus beneficios para la dinamización de la economía de la zona de estudio. Comprobándose que los turistas que visitan el canto Pedernales se sienten atraídos por los locales polifuncionales, demostrándose que el proyecto es económicamente factible y la inversión se recupera a los 3 años, 1mes y un día.

Palabras claves: Estudio de Mercado, Servicio, Evaluación financiera, turismo.

\section{Abstract.}

Study for the creation of a multifunctional restaurant belonging to the touristy sector as a dynamic alternative of the economy in the region of Pedernales. A study on the multifunctionality of the touristy and recreational facilities in the region of Pedernales is made, which includes proposals that incorporate multiple services in one same area. Marketing is developed in a first phase, which enables to determine the demand and prospect of the touristy offer for the project, on the basis of the implementation of

\footnotetext{
${ }^{1}$ Universidad Laica Eloy Alfaro de Manabí. Manabí, Ecuador. escuchef@hotmail.com

${ }^{2}$ Universidad Laica Eloy Alfaro de Manabí. Manabí, Ecuador. evelinalcivar@hotmail.com
} 
instruments that collect information applied to clients. The organizational and administrative analysis, as well as the internal structure of the multifunctional area is itemized prior to the definition of the financial aspects. The total amount of the appropriate investment for the implementation of the study was determined by the financial evaluation, and so were determined the financial indicators, showing the economic feasibility of the project and its benefits to dynamize the economy in the area of study. It was proved that tourists that visit the region of Pedernales are attracted by the multifunctional facilities, and was demonstrated that the project is economically feasibly and the investment is recovered in 3 years 1 month and 1 day.

Keywords: Marketing, Service, Financial evaluation, Travel.

\section{Introducción}

El cantón Pedernales es uno de los cantones perteneciente a la provincia de Manabí que realza los servicios turísticos que se ofrecen en esta región de la costa. Funge como cantón desde el año 1992 y su territorio abarca un área de 1460,7 km2; además, por su sector costero pasa la línea Equinoxial. El atractivo fundamental de este territorio radica en la serenidad de sus playas que tienen una amplitud lo suficientemente extensa para degustar con tranquilidad del mar, unido a hermosas palmeras que están presentes por toda la costa playera, predominando la modalidad del turismo Sol y Playa. Además, esta zona se caracteriza por su rica gastronomía que se destaca en los diversos platos de mariscos que son de alta atracción por los turistas.

En el estudio se interactúa con un antecedente que marca de forma negativa la historia de Pedernales, y son los hechos acontecidos el 16 de abril del 2016, cuando ocurrió un sismo de alta intensidad que afectó desmesuradamente todo el cantón, donde hubo cientos de fallecidos, unido a otro sin número de personas afectadas que se esfuerzan por sacar adelante el cantón. Este fenómeno generó una situación desfavorable para los ciudadanos del lugar, la mayoría son pescadores y emprendedores que su principal fuente de ingreso estaba asociada al turismo local.

Es oportuno agregar que revisando la información asociada con estudios en la zona seleccionada, la mayoría coincide con el perfil turístico y la explotación del mismo, como por el ejemplo el estudio realizado por (Doumet, 2015) pero ninguno de los estudios citados y otros previos ya revisados en la localidad de investigación está asociado a la proyección e implementación de restaurantes polifuncionales como se proyecta en el presente trabajo; aspecto que lo distingue con relación a estudios anteriores.

A raíz de esto surge la necesidad de restablecer las ofertas turísticas de este balneario emblemático tanto para el turismo nacional como internacional, de forma tal que garantice un desarrollo económico para los habitantes de esta localidad. El gobierno nacional está apoyando económicamente los proyectos que contribuyan a realzar el turismo del cantón Pedernales, de forma práctica, eficiente y eficaz. (MIES, 2016). Uno de los elementos que genera este estudio radica en proponer una alternativa de solución a esta problemática, por lo cual surge la siguiente interrogante como problema de investigación:

¿Cómo contribuir a la dinamización de la economía en la zona cero del terremoto del cantón Pedernales? 
La alternativa de solución que se propone se sustenta plenamente con algunos de los objetivos del Plan Nacional para el Buen Vivir (PNBV), según SENPLADES (2013), por ejemplo con el objetivo 5 que plantea "la necesidad de construir espacios de encuentro común y fortalecer la identidad nacional, las identidades diversas, la plurinacionalidad y la interculturalidad"; debido a que con el restaurante polifuncional se desea lograr un espacio con ofertas gastronómicas que vayan más allá de disfrutar un rico menú sino también poder recrear de forma simultánea en el mismo espacio.

También el proyecto se identifica con el objetivo 8 que plantea "consolidar el sistema económico social y solidario de forma sostenible", debido a que la intención esencial de la investigación parte de la utilización de las potencialidades turísticas de la localidad de estudio para el desarrollo de la economía social, involucrando e integrando todos los factores posibles asociados a similares tendencias turísticas.

Otro de los objetivos del PNBV que con que cumple el estudio es el objetivo 9, que nos indica "garantizar el trabajo digno de todas las formas"; el objetivo fundamental del estudio es la dinamización de la economía a partir de la fuente de empleos que generen ingresos en los habitantes de la localidad de estudio de forma honesta.

Estos criterios se resumen en lo que plantea la Normativa Técnica Ecuatoriana, (NTE INEN 2631:2012), donde expresa que: "el desarrollo turístico en el litoral ecuatoriano debe ser sustentable y consciente, para evitar la degradación progresiva de sus principales atractivos naturales y culturales, prevenir problemas derivados del deterioro ambiental, para que las futuras generaciones se beneficien de esta actividad, en conformidad con lo establecido en el régimen del Buen Vivir establecido en la Constitución del Ecuador”.

En función de estos hechos muchas de las ofertas gastronómicas, turísticas y de los servicios recreativos nocturnos para turistas se vieron afectados, aspectos que justifica la necesidad de rescatar estas ofertas turísticas, por tal razón se realiza el estudio de investigación asociado a la polifuncionalidad gastronómica y recreativa para fortalecer la economía de esta región devastada.

Es necesario agregar que la competitividad en el sector gastronómico turístico facilita la diversificación de ofertas para los turistas, generando una mayor expectativa y demanda de los clientes para la contribución al desarrollo de la economía con la creación de nuevos empleos y del uso eficiente de los medios turísticos, de forma tal que se reducen costos y se maximizan el uso de las instalaciones turísticas.

Realizar un estudio de factibilidad para la creación de un restaurante polifuncional del sector turístico como alternativa dinamizadora de la economía en la zona cero, del cantón Pedernales.

$>$ Definir a través de un estudio de mercado las expectativas que tienen los turistas con respecto al servicio de restaurantes polifuncionales en el balneario turístico del cantón Pedernales.

$>$ Determinar a través de un estudio técnico el tamaño, la localización y los recursos necesarios para la puesta en marcha del proyecto propuesto.

$>$ Diseñar la estructura organizacional y administrativa turística que permitan la ejecución del proyecto con las competencias necesarias e indispensables para su funcionamiento. 
$>$ Evaluar la vialidad del proyecto a través de un estudio financiero que permita la toma de decisiones sobre su implementación.

\section{Materiales y métodos.}

\section{El turismo como dinamizador de la economía}

En las últimas décadas el turismo se ha mostrado como una actividad con potencial para diversificar la estructura económica de los países frente a actividades económicas tradicionales. (Leader, 2011). Ecuador, un país con una estructura productiva muy dependiente del sector primario, con la necesidad de diversificar su matriz productiva y generar nuevos ingresos que mejoren su economía, ha impulsado una serie de iniciativas en el sector turístico.

En los últimos años se mantiene una tendencia económica creciente, importante en la región, evidenciada en el crecimiento de su producto interno bruto (PIB). El crecimiento económico del Ecuador se debe a una serie de decisiones importantes sobre los generadores de ingresos económicos, pasando de ser un país enfocado en el sector primario a desarrollar el sector industrial y sobre todo el sector de los servicios. (MINTUR, 2016).

Según MINTUR (2016) los ingresos del turismo en el PIB del Ecuador en los últimos años va en crecimiento, aspecto que muestra el dinamismo que ha generado para la economía nacional las inversiones realizadas por parte del gobierno orientada a la promoción de los productos turísticos del país.

El sector turístico en Pedernales está marcado por su privilegiada geografía, con una extensión costera que llega a los $54 \mathrm{~km}$ de playa, es atractivo de la mayoría de los turista que la visitan, haciendo del lugar un centro turístico de gran proyección, con una facilidad de acceso producto de la nueva infraestructura vial, que conectan al Cantón con las ciudades de alta población como Quito, Guayaquil y Santo Domingo. (GAD, 2015).

Actualmente existe una extensa y variada infraestructura turística, hay hoteles para todos los gustos y presupuestos, llegando a casi los 50 establecimientos para alojamiento desde cuarta categoría hasta uno de 4 estrellas, de la mano de personal profesional y capacitado. De estos establecimientos 18 están dentro del programa solidario para el turismo que se elaboró en el 2016 producto de los acontecimientos ocurridos con el terremoto.

Existe una proyección en el cantón pedernales de forma tal que la actividad turística llegue a ser el segundo sector productivo primario y se faciliten la distribución de las riquezas que se obtienen del turismo. Además, las autoridades deben regular la actividad de forma tal que se preserve el recurso panorámico natural y evitar el deterioro por las sobrecarga turística, en fin promover un turismo consiente y sustentable.

\section{Estudio de mercado}

\section{Estudio de campo}

El trabajo de campo implica la selección, capacitación y supervisión de las personas que reúnen los datos para ser procesados. (DRH, 2011). Para la investigación se recopiló información en el cantón de Pedernales, específicamente en la cabecera cantonal Pedernales y en la parroquia rural Cojimies, dado que son las localidades de mayor 
asistencia y movimiento turístico, así como en la ciudad de Santo Domingo, dado que sus pobladores visitan frecuentemente las playas de Pedernales por su cercanía.

\section{Población}

La población considerada para el estudio como referencia no se precisa con exactitud dada la falta de fiabilidad de las fuentes que registran la media de los turistas que asisten al cantón en estudio. Con esta consideración se determinó la muestra de estudio a partir de una población desconocida, con un índice de confianza de un 95\% y con un valor de significancia de 0.05 , por lo que el coeficiente de 1,96 establece la distribución nominal de la muestra, que se calcula con la siguiente fórmula:

$$
n=\frac{Z a^{2} * p * q}{E^{2}}
$$

Dónde:

\begin{tabular}{ccc} 
Símbolo & Concepto & Datos \\
\hline $\mathrm{n}$ & $\begin{array}{c}\text { Tamaño de la muestra } \\
\mathrm{Za}\end{array}$ & Referencia Estandarizada con un nivel de confianza \\
$\mathrm{E}$ & superior 90\% & 1.96 \\
$\mathrm{p}$ & Error máximo permisible & $5 \%$ \\
$\mathrm{q}$ & Probabilidad de Ocurrencia & $50 \%$ \\
& Probabilidad de No Ocurrencia (p-1) & $50 \%$
\end{tabular}

Se consideró trabajar con un error máximo permisible del 5\% para mantener un índice de confianza favorable para el estudio, superior al $90 \%$. El resultado nos indica un tamaño maestral de 384 elementos.

La encuesta se aplicó a la población económicamente activa que realiza turismo con frecuencia en la zona de estudio. Se visitó a los propietarios de las cabañas en la zona playera de Pedernales y a los dueños de los restaurantes cercanos a la playa en Cojimies. Se aplicó la encuesta a los 384 turistas y a 6 de los 14 propietarios de cabañas y restaurantes con las características para implementar el estudio.

\section{Diseño de los instrumentos}

Los instrumentos que se aplican se diseñaron con la intención de recopilar información relacionada con el nivel de aceptación que ofrece la implementación de un servicio de múltiples ambientes en horarios distintos, consta de 18 interrogantes de la cuales, hay 5 que se relacionan directamente con la factibilidad de la propuesta, las cuales fueron analizadas bajo el criterio actitudinal de evaluación de la escala Likert. (Malhotra, 2008). Las entrevistas realizadas constan de 18 preguntas que tienen la intencionalidad de captar la valoración respectiva por parte de los propietarios de los locales sobre la intencionalidad del proyecto. Son preguntas directas y abiertas que en su mayor interés profundiza en la disponibilidad de los propietarios para implementar el proyecto.

\section{Demanda}

La demanda del servicio se determina a partir de la magnitud de la asistencia turística a la zona de estudio, considerándose también la segmentación aplicada en el mercado. 
Revisando los datos obtenidos por el estudio de mercado, se percibe que el cantón Pedernales es visitado por turistas de diversas zonas del Ecuador, donde la cantidad mayoritaria es de la Costa y de Sierra, destacándose la población de Santo Domingo de los Tsáchilas que tiene una alta afluencia turística a la zona de estudio. Es importante destacar que no existe un conocimiento de los locales polifuncionales, el $43 \%$ no los conoce; por su parte cuando el $44 \%$ al conocer las características de los locales polifuncionales lo consideran muy atractivo, incluso el $73 \%$ de los turistas se motivan por la oferta gastronómica y los bares en sus viajes turísticos.

\section{Análisis de demanda}

Para el estudio de la demanda se partió de la información obtenida por el catastro del Ministerio de Turismo y el GAD de Pedernales, donde se indican los visitantes del año 2013, lo cual requiere una proyección de demanda para los años venideros que se ejecutará la propuesta. Por lo tanto a partir de los datos obtenidos, se proyecta desde el 2014 para 5 años la demanda futura, con una tasa de crecimiento de $1.37 \%$, que se ajusta a la tasa de crecimiento poblacional para Ecuador a partir del 2014 según el Instituto Nacional de Estadísticas y Censos (INEC).

Tabla 1: Proyección de la demanda

\begin{tabular}{cc}
\hline Años & Turistas \\
\hline 2014 & 42685 \\
2015 & 43270 \\
2016 & 43862 \\
2017 & 44463 \\
2018 & 45073 \\
2019 & 45690 \\
2020 & 46316 \\
\hline
\end{tabular}

Fuente: Propia (2017).

\section{Oferta}

A partir de los resultados obtenidos en la demanda proyectada se procede a la determinación de la oferta, que serían aquel segmento de turistas a los que va dirigido el producto. Actualmente en el cantón de Pedernales no existe este tipo de producto con las características como se está proyectando.

\section{Análisis de oferta}

Considerando los resultados obtenidos en las encuestas aplicadas el $49 \%$ de los encuestados encuentran atractivo el local polifuncional como alternativa de recreación para disfrutar de sus servicios. Existen 7 locales con características similares al de estudio, conocidos como caipiriñas, que actualmente no brindan el servicio solicitado, por lo tanto para definir la oferta se tomará en cuenta el $49 \%$ de la demanda proyectada; y de ese porcentaje se le hallará el 14,3\% que equivale a 1 de los 7 locales similares y será la capacidad de la oferta que generará el bar- restaurante Aventura que es tomado como referencia en el estudio, lo que a modo de resumen significa que la oferta será el $7 \%$ de la demanda proyectada. 
En la Tabla 2 se proyecta la oferta siguiendo estos parámetros por el nivel de competitividad en el mercado, este tipo de negocios aplica con fuerza el benchmarking y suelen implementar de forma inmediata servicios similares.

\section{Demanda insatisfecha}

Para determinar la demanda insatisfecha se procede a determinar la demanda que no ha sido cubierta en el mercado y que puede ser cubierta. En la Tabla 2 se muestra la demanda insatisfecha.

Tabla 2: Proyección de la oferta y demanda insatisfecha proyectada.

\begin{tabular}{cccc}
\hline Años & Demanda & Oferta & $\begin{array}{c}\text { Demanda } \\
\text { Insatisfecha }\end{array}$ \\
\hline 2016 & 43862 & 3073 & 40789 \\
2017 & 44463 & 3116 & 41348 \\
2018 & 45073 & 3158 & 41914 \\
2019 & 45690 & 3201 & 42489 \\
2020 & 46316 & 3245 & 43071 \\
\hline
\end{tabular}

Fuente: Propia. (2017).

Como se percibe en la tabla la demanda insatisfecha equivale al $92 \%$ de la demanda del mercado, por lo tanto según estos datos se puede considerar un alto déficit en la satisfacción de las necesidades del cliente, por lo cual los servicios gastronómicos son menores a los requerimientos de los clientes.

\section{Oferta del producto}

Dado la inestabilidad turística del cantón de estudio y conociendo que los periodos de mayor demanda son los feriados, se procede a determinar la oferta del producto a partir del conocimiento de que existen 4 meses en el año que son de ventas bajas por la poca afluencia de turista, por los tanto se procede a distribuir la oferta entre los 8 meses restantes del año que más movimiento de turistas existen, lo que genera una oferta de producto promedio para 395 clientes por mes. Es importante destacar que en los días feriados esta cifra crece exponencialmente.

En la Tabla 3 se muestra como la oferta del producto mensual equivale al $1 \%$ de la demanda anual de clientes, aspecto que indica que existe un mercado amplio para trabajar esa y mejorar la oferta del producto.

Tabla 3: Oferta del proyecto

\begin{tabular}{cccc}
\hline Años & $\begin{array}{c}\text { Demanda } \\
\text { Anual }\end{array}$ & $\begin{array}{c}\text { Oferta } \\
\text { Mensual }\end{array}$ & $\%$ \\
\hline 2016 & 43862 & 384 & 1 \\
2017 & 44463 & 389 & 1 \\
2018 & 45073 & 395 & 1 \\
2019 & 45690 & 400 & 1 \\
2020 & 46316 & 406 & 1 \\
\hline
\end{tabular}

Fuente: Propia. (2017). 


\section{Mix de marketing}

Los locales polifuncionales establecerán estrategias para alcanzar los objetivos propuestos y dinamizar la economía, satisfaciendo a sus clientes a través de la aplicación de un mix de marketing enfocado en el producto, precio, plaza y promoción; de forma tal que garantice las condiciones estratégicas para el desarrollo de la propuesta.

\section{Producto}

Existen siete locales bares-restaurantes que cumplen con los requisitos espaciales y técnicos para implementar la propuesta, los cuales son Salsa Coctel, El emigrante, Caída del Sol, Bar-Dudu, Parales, Son Candela y Aventura. De los mismos se utiliza como referencia para el estudio el bar-restaurante "Aventura", que ofrece servicios de recreación con una mayor actividad comercial vespertina nocturna, orientada más al servicio de restaurantes con platos a la carta y algunas bebidas típicas de la competencia en la zona.

La intención fundamental del producto a implementar es la incorporación de servicios gastronómicos en la mañana, además de diversificar la oferta recreativa de bar nocturna con la integración de actividades bailables organizadas, de forma tal que el establecimiento esté activo brindando servicio desde la $07 \mathrm{H} 00$ hasta las $02 \mathrm{H} 00$, sobre todo en los feriados.

Por lo que el servicio gastronómico propuesto para el horario matutino, no estará determinado solamente por platos a la carta, sino que se desea incorporar una especie de mesa buffet con la perspectiva de darle mayores alternativas al cliente en el menú y a su vez permite optimizar el personal de trabajo.

\section{Marca}

Se sugiere mantener la misma marca del local, pues tiene posicionamiento y fidelización de algunos clientes, además de ser una propuesta condicionante del propietario.

Para un mejoramiento en la imagen y el mensaje se procede a incorporar dentro de la marca ilustraciones de cocteles que favorecen e identifican el mensaje de los productos que se ofrecen; además, se agrega como slogan para la nueva funcionalidad del local polifuncional el siguiente: "Fiesta y paladar, el placer de la experiencia..."; dado que con esta frase se hace referencia a lo que se ofrece dentro del local y su carácter integrador de servicios.

\section{Precios}

Los precios que se proponen se manejan de acuerdo con la competencia, pues existe un acuerdo entre los comerciantes de los locales de no hacer competencia de precios. Por lo tanto se manejan los precios por plato de acuerdo a lo convenido por los comerciantes. Dado la necesidad de activar la actividad matutina a través de un servicio de mesa buffet para los desayunos y servicios gastronómicos del local, se muestra en la Tabla 4 las tarifas de ofertas que se brindan según los precios promedios y la competencia. 
Tabla 4: Tarifas de Precios

\begin{tabular}{lccc}
\hline Servicios & $\begin{array}{c}\text { Precio } \\
\text { Competencia } \\
\text { (USD) }\end{array}$ & $\begin{array}{c}\text { Precio } \\
\text { "Aventura" } \\
\text { (USD) }\end{array}$ & $\begin{array}{l}\text { Variación } \\
\%\end{array}$ \\
\hline Desayunos & 3.00 & 2.75 & $-8.3 \%$ \\
Almuerzos & 4.50 & 3.50 & $-22 \%$ \\
$\begin{array}{l}\text { Platos a la } \\
\text { Carta }\end{array}$ & 6.00 & 6.00 & $0 \%$ \\
Bebidas & 2.00 & & \\
Karaoke & 0.25 & 1.50 & $-25 \%$ \\
\hline
\end{tabular}

Fuente: Propia. (2017)

Como se percibe, la oportunidad de brindar un servicio de mesa buffet permite reducir costos, lo cual facilita una estrategia de precios para destacar el producto que se ofrece.

\section{Ubicación del local}

La ubicación del local polifuncional está en un área de bastante afluencia de turistas, en plena playa de pedernales, lo cual facilita la interacción directa del consumidor con el producto que se le ofrece. En dicho lugar asisten comerciantes de mariscos y turistas con la intención de consumir un desayuno, acción que sugiere y justifica la alternativa de incorporar actividades gastronómicas en las mañanas.

\section{Promoción}

Para el proceso de promoción se parte de aplicar una estrategia comunicacional centrada fundamentalmente en el uso de las redes sociales, canales de radio y televisión. De las herramientas más favorables y con costos factibles de promoción se procede por utilizar en primera instancia la red social Facebook; se decide utilizar en primera instancia este canal dado el alcance que tiene en las comunicaciones pues las cantidad de personas que interactúan con la red son mayores, además se captan comentarios de los internautas que sirven para reflexionar sobre las ofertas que se diseñan.

También se proyecta el uso de los canales de radio con una frecuencia de 2 a 3 veces a la semana, seleccionando preferiblemente Radio Altamar de Pedernales, Radio Mega Estación de Santo Domingo, Radio Zaracay de Santo Domingo y Radio Canela de Quito. A su vez se prevé incursionar en los canales televisivos con una frecuencia de 1 a 2 semanal, enfocándose principalmente en Zaracay TV de Santo Domingo, Majestad TV de Santo Domingo y a nivel nacional en Tele Amazonas y Ecuador TV.

Para el diseño del Spot publicitario se considera incorporar un mensaje que manifieste lo que se comunica en el slogan, donde quede claro la polifuncionalidad del local. Además de utilizar banners publicitarios para promocionar las ofertas que tiene el restaurante polifuncional.

Como se percibe existen elementos publicitarios que facilitan la promoción de la oferta para los turistas y la posición de los locales permite una publicidad en el punto de venta que facilita la atracción de los clientes de forma directa. 
Es importante destacar que en este tipo de negocios la publicidad del boca a boca a través del marketing directo es la más efectiva, pues se observó en el estudio de mercado que los clientes se identifican con las sugerencias de amistades o familiares cuando realizan actividades turísticas.

\section{Estudio técnico}

En el proceso de valorar la factibilidad del proyecto se consideró en el estudio técnico la extensión superficial del local, el cual ocupa un área aproximada de $218 \mathrm{~m} 2$, con una capacidad para 120 personas que pueden desplazarse libremente sin congestión en el mismo.

\section{Localización del proyecto}

Uno de los aspectos más importante del proyecto es la localización del local, este se encuentra ubicado en el cantón Pedernales de la provincia de Manabí. El estudio de la localización tanto macro como micro facilita el análisis de variables que pueden incidir de forma activa en el proyecto propuesto.

\section{Microlocalización}

El local se encuentra ubicado específicamente en el malecón de pedernales, siendo parte de los famosos llamados caipiriñas, donde los turistas disfrutan de bebidas agradables en los ambientes de playa.

\section{Ingeniería del proyecto}

Para la ingeniería del proyecto se parte de la identificación de los dos ambientes esenciales en los que se sustenta la polifuncionalidad. El ambiente para el horario matutino y el ambiente para el horario vespertino y nocturno.

\section{Ambiente de servicios}

Se analizó la distribución de las áreas con las zonas públicas y privadas dentro del local, así como por cada ambiente, la función, actividad y mobiliario que se necesita y se tiene para la atención al cliente de forma efectiva. Se desarrolla un cuadro de actividad por ambiente que facilita la comprensión de las funciones y actividades que se van a realizar por cada zona:

$>$ Restaurante (A1): Ofrecer el confort necesario para los servicios gastronómicos de alimentación en la mesa buffet y los platos a la carta (pasa a ser un área bailable en el horario nocturno).

$>\operatorname{Bar}(\mathrm{A} 2)$ : Brindar un servicio de calidad, incorporando criterios de enología en los tragos y licores.

$>$ Juegos infantiles (A3): Generar el espacio necesario para la recreación sana de los clientes menores de edad.

$>$ Área pasiva (A4): Ofrecer un espacio para que los clientes se recreen de forma pasiva.

$>$ Caminerias (A5): Facilitar el acceso al local desde sus alrededores y hacia la playa.

$>$ Áreas verdes (A6): Ornamentar y recrear el local. 
$>$ Playa (A7): Recrear al cliente en su interacción con el mar y la arena como recursos naturales.

$>$ Cocina (A8): Confeccionar los alimentos para los clientes.

$>$ SSHH (A9): Facilitar el uso de sanitarios para los servicios de baño para ambos sexos.

$>$ Caja (A10): Cobrar y archivar los recursos monetarios del local.

Diseño del local

El local del restaurante polifuncional está ubicado en la vía principal del malecón de Pedernales, limitado por otros locales similares conocidos como caipiriñas, el mismo está divido en tres áreas fundamentales, como se muestra en la Figura 1, el área de baños y lavabos, el área de cocina y el área más amplia que es donde está ubicado el bar con la mesa buffet y las 10 mesas disponibles para brindar un servicio de calidad. Se cuenta con dos televisores Samsung de 40 pulgadas para la visualización de programas, a su vez en la noche se activa un proyector donde se pasan videos clic de cantantes y músicos de diversos géneros para la satisfacción de los clientes. El local cuenta con dos vías de acceso, una hacia la vía del malecón y la otra hacia la playa, y tiene un servicio de telefonía para llamadas locales que está a disposición de los clientes. Para el diseño del local se tuvieron en cuenta aspectos de organización planteados por (Boullon, 2003) (Villena, 2008).

Figura 1. Diseño de la planta del local polifuncional

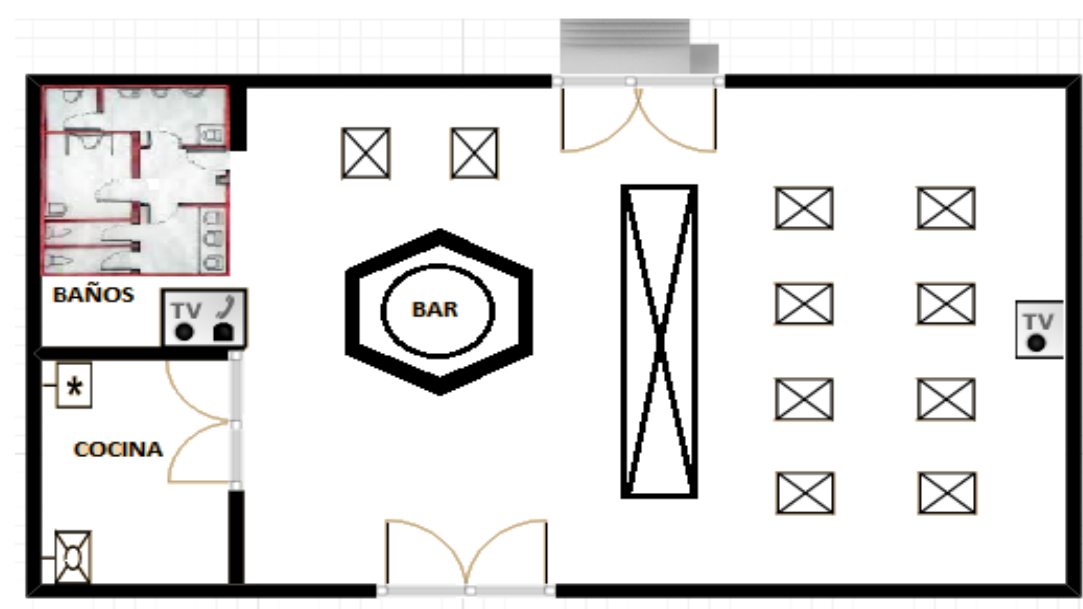

Fuente: Propia. (2017).

\section{Elaboración del menú}

Las ofertas son estándar diseñada para un promedio de clientes en periodo de no feriado, para los periodos de feriado se incrementa el menú y se diversifica.

El propietario del negocio cuenta con diversas empresas, como camaroneras y fincas en el cantón de Santo Domingo de los Tsáchilas que favorecen el abastecimiento de los suministros de la materia prima para la elaboración de alimentos. Los proveedores que abastecen los productos del mar al establecimiento son de la empresa de pesca del propietario del negocio. Las bebidas y licores se adquieren a través de un acuerdo que se 
tiene con una comercializadora mayorista de la ciudad de Guayaquil que abastece quincenalmente el local. Es importante destacar que la relación del propietario con los proveedores es duradera pues el negocio lleva más de 10 años en el mercado, con registro único del contribuyente desde el 2006.

Para lograr resultados satisfactorios es necesaria la calidad del servicio en el desempeño de las operaciones; este se fundamenta en la profesionalidad con que se brindará el mismo, tomando en consideración los estándares de calidad. Para eso, el bar restaurante como parte de la gestión operativa les dará la oportunidad a los clientes de que emitan criterios sugerentes para el mejor desempeño de las operaciones.

Para los clientes en la zona de playa el uso adecuado del tiempo es fundamental, uno de los aspecto que favorece la optimización del tiempo para ellos es la ventaja que ofrece la mesa buffet, pues limita el tiempo de espera y les permite disfrutar del servicio solicitado con la mayor prontitud posible.

Actualmente una preocupación de los clientes y de los organismos de sanidad es la prevención de riesgos por manipulación inadecuada de los alimentos, por lo que en el local se maneja las normas elementales de higiene y manipulación de los alimentos, fundamentalmente en los mariscos que requieren el cumplimiento de normas cuidadosas para su uso.

La empresa desde el punto de vista legal está debidamente constituida, con un Registro Único de Contribuyente (RUC) establecido desde 2006 para realizar sus declaraciones y con los permisos y licencias actualizados año por año para su debido funcionamiento.

\section{Estudio financiero.}

Las proyecciones económicas y financieras para evaluar la factibilidad de implementar el proyecto se realizan teniendo en cuenta los requisitos legales establecidos al respecto y empleando software profesional como el Microsoft Excel. Para la aplicación del proyecto se analizan y realizan inversiones de activos, los costos de mano de obra, los costos unitario de la materia prima, los costos unitario de materiales indirectos, los costos de servicios básicos y gas, imprevistos y depreciaciones.

\section{Resultados y discusión.}

\section{Resultados de la encuesta aplicada}

Pregunta 1.- ¿Es usted de la región?:

Interpretación y análisis: El 56\% de los encuestados pertenecen a la región Costa y el $43 \%$ corresponden a la región de la Sierra. Un 1\% son de la Amazonía y Oriente y no se encuestó a ninguna persona del exterior. El Ecuador se divide en cuatro importantes regiones, pero queda claro que la mayor parte de los encuestados son de la región Costa, otra parte significativa son de la Sierra, no siendo así con los de la Amazonía, ni con los del Oriente, lo que muestra donde está ubicada nuestro segmento de interés para el desarrollo del proyecto.

Pregunta 2.- ¿Considera las playas de Manabí un lugar atractivo para hacer turismo? 
Interpretación y análisis: El 88\% de los encuestados consideran las playas de Manabí un lugar atractivo para hacer turismo, el 12\% restante no está de acuerdo con la afirmación. Por lo que para el proyecto este nivel de aceptación es muy favorable pues consolida el punto de venta para la implementación de la propuesta.

Pregunta 3.- ¿Con qué frecuencia visita la playa de Pedernales para su recreación?

Interpretación y análisis: El 66\% de los encuestados afirman que visitan las playas de Pedernales solo en días feriados, el $13 \%$ las visita cada dos meses, el $7 \%$ dos veces al mes, un $5 \%$ afirman que casi nunca y solo el $4 \%$ las visita una vez al mes. Esta información indica que el momento para fidelizar los clientes y darle mayor publicidad al proyecto es en feriado.

Pregunta 4.- Cuando visita las playas de la costa aparte de deleitarse con el balneario, ¿qué otras preferencias turísticas disfruta?: (Señale las tres que más prefiere)

Interpretación y análisis: El 51\% prefiere las ofertas gastronómicas cuando visita alguna playa, el $23 \%$ prefiere los bares, un 17\% le atraen más las excursiones y al 10\% les gustan las fiestas nocturnas. Sin lugar a dudas, las ofertas gastronómicas son las que tiene mayor preferencia por los turistas que visitan las playas, este aspecto indica que el proyecto de polifuncionalidad tendrá interés en los turistas dado su nivel de preferencia.

Pregunta 5.- Cuando está de visita en la costa ¿qué tipo de oferta gastronómica prefiere?:

Interpretación y análisis: el $66 \%$ de los encuestados prefieren la comida de la costa, otro dato significativo es que el $30 \%$ prefiere los platos a la carta, solo el $1 \%$ tiene preferencia por la comida serrana y a un $3 \%$ les gusta la comida gourmet. Aspectos estos importantes para la oferta del menú a proponer en los locales del proyecto.

Pregunta 6.- ¿Qué nivel de atracción le genera un local que le ofrezca servicios gastronómicos por el día y actividad festiva en las noches?

Interpretación y análisis: El 44\% de los encuestados les resulta muy atractivo, el $28 \%$ lo ven atractivo, el $27 \%$ no les atrae, pero tampoco les desagrada, y solo a un $1 \%$ les resulta poco atractivo. Evidentemente los lugares que ofrezcan servicios gastronómicos por el día y actividad festiva en las noches les resultan favorables, aspecto que es esencial para el proyecto por la novedad de la propuesta.

Pregunta 7.- ¿En qué horario del día le resulta más satisfactorio visitar los bares y los restaurantes?

Interpretación y análisis: Un $27 \%$ de las personas encuestadas tiene preferencia por asistir en horario de la noche, el $24 \%$ desea asistir al medio día, otro $24 \%$ los elige en cualquier horario del día, el 16\% tiene inclinación por el horario de la tarde, y un $9 \%$ opta asistir en la mañana. Claramente la mayor parte de los encuestados prefiere el horario de la noche para asistir a bares y restaurantes, elemento que ayuda a definir estrategias fundamentales para la satisfacción del cliente.

Pregunta 8.- ¿Cuál es el tipo de establecimiento recreativo que usted más frecuenta normalmente? 
Interpretación y análisis: El 29\% de los encuestados prefieren los restaurantes para pasar un rato agradable y de recreación, el $22 \%$ prefieren las discotecas, el $21 \%$ elige los bares, otro $20 \%$ opta por los cines, solo el $4 \%$ selecciona los clubes y otro $3 \%$ escoge otros lugares para su recreación. Esto ayuda a definir las funciones más atractivas para la funcionalidad del establecimiento.

Pregunta 9.- Existen en su localidad centros que brinden servicios de múltiple ambiente en horarios distintos del día (restaurante/bar/cafetería/club nocturno).

Interpretación y análisis: El 43\% de los encuestados afirman que no conocen la existencia en su localidad de centros que brinden servicios de múltiple ambiente en distintos horarios del día. El 33\% atestiguan que sí existen estos tipos de locales y el $24 \%$ dicen que no existen. Se evidencia que no conocen o no existen muchos centros que tengan múltiples ambientes en distintos horarios del día.

Pregunta 10.- Has frecuentado alguno de estos centros.

Interpretación y análisis: El $67 \%$ de las personas encuestadas no han asistido nunca a centros que brinden servicio de ambiente múltiple y el 33\% afirman si haber asistido. Queda claro la poca existencia o el poco conocimiento sobre este tipo de centros en su localidad.

Pregunta 11.- Si existiera en los balnearios centros con múltiples ambientes en un mismo local en horarios distintos, ¿los visitaría?

Interpretación y análisis: El 100\% de las personas encuestadas afirman que si visitarían estos locales.

Pregunta 12.-. ¿Cuáles son las razones de su preferencia por las que asistiría a estos locales?

Interpretación y análisis: El 25\% de los encuestados afirman que la razón por la que más prefieren asistir a lugares recreativos o de múltiple ambiente es para comer, el $22 \%$ dicen que por beber, el $20 \%$ lo hacen por conocer personas, el $16 \%$ para escuchar música, un $10 \%$ se inclina por el baile y otro $5 \%$ por disfrutar de shows.

13.- ¿Que amenidades le gustaría encontrar en un local con múltiples ambientes en horarios distintos?

Interpretación y análisis: La amenidades que más prefieren las personas está la de escuchar una banda u orquesta en vivo, con $31 \%$ de preferencia por parte de los encuestados. Los concursos y competencias tuvieron $29 \%$ de preferencia. Juegos y entretenimientos con $21 \%$ de predilección.

Pregunta 14.- ¿Qué servicios motiva su preferencia como cliente de estos locales polifuncionales?

Interpretación y análisis: De los servicios brindados en locales polifuncionales el que más motiva a los clientes son los precios y variedad del servicio con $32 \%$ de preferencia, la ubicación del local con $22 \%$, el ambiente con $12 \%$ y otras preferencias con $1 \%$.

Pregunta 15.- En qué zona le resulta más favorable la existencia de este tipo de locales que oferte múltiples ambientes en horarios distintos: 
Interpretación y análisis: La zona de mayor preferencia para la existencia de locales polifuncionales es cerca de la playa con $34 \%$ de aceptación, le sigue zonas residenciales y tranquilas con $33 \%$, el centro de la ciudad con $18 \%$, el $13 \%$ para las afueras de la ciudad, y las zonas rurales con el mínimo de preferencia de $1 \%$.

Pregunta 16.-. ¿Cuándo asistes a algún centro recreativo, cuánto sueles gastar aproximadamente?

Interpretación y análisis: La mayor parte de los encuestados gastan aproximadamente de 101 a 300 USD, ya que este aspecto obtuvo un 35\% de marcación. El 25\% gasta un promedio de 300 a 500 USD, un $17 \%$ consume entre 50 a 100 USD. Aspecto de vital interés para la proyección de ofertas y precios atractivos dentro del proyecto propuesto.

Pregunta 17.- ¿Cuando sale a algún centro recreativo lo hace acompañado?

Interpretación y análisis: El 33\% de las personas encuestadas afirman que cuando asisten a algún centro recreativo lo hacen acompañado de su pareja, el $27 \%$ alega que lo hace con amigos, un $24 \%$ lo hace en familia y solo el $15 \%$ acude a estos centros solo.

Pregunta 18.- Recomendaría a sus allegados la visita a un local con múltiples ambientes en horarios distintos.

Interpretación y análisis: Todos los encuestados (100\%) recomendarían este tipo de sitio polifuncional a sus allegados. Los establecimientos que brindan servicio de múltiple ambiente en distintos horarios del día son muy atractivos para la diversión y el esparcimiento.

De manera general se puede concluir que más de la mitad (66\%) tiene una alta preferencia por visitar las playas de pedernales en feriado, a su vez el $73 \%$ de los turistas se sienten motivados por la oferta gastronómica, lo cual es satisfactorio pues significa que existe una demanda significativa de servicios gastronómicos en las preferencias de los turistas; a su vez el $49 \%$ de los encuestados se sienten muy atraídos por los locales polifuncionales como alternativa gastronómica y recreativa.

\section{Resumen de costos y gastos para la prestación de servicio}

En la Tabla 5 se resumen todos los costos directos e indirectos que intervienen en el proyecto, así como su comportamiento en los primeros 5 años de ejecución.

Tabla 5: Costos y gastos del proyecto

\begin{tabular}{cccccc}
\hline \multirow{2}{*}{ Costo/Periodo } & \multicolumn{5}{c}{ Años del proyecto } \\
& 1 & 2 & 3 & 4 & 5 \\
\hline Costos directos de & 163 & 166 & 169 & 173 & 176 \\
producción (USD) & 327.15 & 593.69 & 925.57 & 324.08 & 790.56 \\
$\begin{array}{c}\text { Costos indirectos de } \\
\text { producción (USD) }\end{array}$ & 1784.48 & 1820.16 & 1856.57 & 1893.70 & 1931.57 \\
Gastos administrativos & 1068.87 & 1068.87 & 1068.87 & 1068.87 & 1068.87 \\
(USD) & 166 & 169 & 172 & 176 & 179 \\
Total (USD) & 180.50 & 482.73 & 851.01 & 286.65 & 791.00 \\
\hline
\end{tabular}

Fuente: Propia (2017). 
Para desarrollar la prestación del servicio en el primer año de operaciones es necesario utilizar 166 180.50USD, con una tasa de crecimiento proyectada del $2 \%$ anual dado la situación actual producto del fenómeno sísmico, ya que los turistas tienen cierta incertidumbre con relación al territorio.

\section{Proyección de ingresos}

Los valores de ingresos producto de la gestión de venta son adquiridos del servicio prestado para la polifuncionalidad del local, partiendo de los horarios de trabajo predefinido, existen tres etapas; la de desayuno con mesa buffet que tiene un horario de 07H00-9H00; la de servicio de restaurante con plato a la carta que tiene un horario de $13 \mathrm{H} 00-19 \mathrm{H} 00$ y el servicio del bar que tiene un horario de 14H00-02H00. Dado la polifuncionalidad se tiene previsto pagar las horas adicionales que sean requeridas por trabajadores para realizar sus servicios.

Existe un margen de utilidad del precio de venta con relación al costo de producción, lo que genera un ingreso diario de 940.00USD por concepto de ventas, que a su vez significa un ingreso mensual de 15 040.00USD y para el año un ingreso de 180 480.00USD; se considera 16 días de trabajo mensual pues es la media de los días en que se ofrecen servicios gastronómicos, según se constató en la investigación de campo.

\section{Estados financieros proyectados}

Se proyecta el resultado de las operaciones para los primeros 5 años con un crecimiento en ventas de un 3\% anual. También facilita la medición del rendimiento financiero de la empresa producto de su actividad comercial, dada la medida del índice de utilidad con relación a las ventas.

Existe un margen de utilidad neta positiva de 10 331.39USD para el primer año que equivale aproximadamente al $5.7 \%$ de las ventas generadas en la prestación de servicios, y se mantiene en ese porcentaje para los restantes periodos. Además, la utilidad bruta de la gestión muestra un valor del 17 152.85USD que equivale al 9.5\% de las ventas.

\section{Flujo de caja proyectado}

En el flujo de caja se percibe como se mueven los fondos líquidos dentro del proyecto, donde se parte de un desembolso de 17 727.44USD y dada la proyección en el primer año no existe una recuperación de la inversión realizada; por lo tanto se requiere de dos años de operaciones como mínimo para la recuperación de la inversión, aspecto que se fundamenta más adelante con los indicadores financieros.

\section{Balance general}

La situación inicial de la empresa (Año 0) parte de la inversión de 17 727.44USD para el desarrollo del proyecto, este monto forma parte de la adquisición de capital producto de las opciones de financiamiento no reembolsable que el Ministerio de Industria y Productividad (MIPRO) le está otorgando a los empresarios en el cantón Pedernales que demuestren proyectos económica y financieramente rentables para la zona. Este monto del MIPRO tiene varias escalas y ofrecen 20 mil a los proyectos de pequeñas y medianas empresas que impulsen el turismo en la zona. 
Los activos se duplican en el primer año que sumaban un monto de 17 727.44USD, para el segundo año alcanzó los 38 778.31USD, por otra parte se refleja la ventaja de no tener obligaciones a raíz de la facilidad que brinda el MIPRO con los créditos no reembolsables.

\section{Evaluación financiera del proyecto}

Se calcula el Valor Actual Neto (VAN) del proyecto a futuro, aplicando una tasa mínima de rendimiento del $6.56 \%$, obtenida a partir de la sumatoria de la tasa de inflación $1.05 \%$ y la tasa pasiva de $5.51 \%$ ambas para noviembre 2016 según el BCE.

$$
V A N=\frac{F o}{(1+i)^{0}}+\frac{F 1}{(1+i)^{1}}+\frac{F 2}{(1+i)^{2}}+\frac{F 3}{(1+i)^{3}}+\frac{F 4}{(1+i)^{4}}+\frac{F 5}{(1+i)^{5}}
$$

El VAN obtenido es de 15 189.38USD, significa que el VAN es mayor que cero, aspecto que es favorable para el proyecto pues indica que es viable el proyecto según las interpretaciones de los indicadores financieros, dado que este índice muestra la afectación del valor del dinero en el tiempo, lo que hace una referencia del comportamiento de los flujos futuros a valor presente.

Después de determinar el valor actual neto del proyecto y ver que el resultado indica la viabilidad del mismo, se procede al cálculo de la tasa interna de retorno.

La tasa mínima atractiva de rendimiento (TMAR) para el proyecto fue de $6.56 \%$ y la tasa interna de retorno (TIR) obtenida es de 30\%, muy superior a la TMAR, estos valores reafirman la viabilidad del proyecto.

Para el cálculo del período de recuperación de la inversión (PRI) se aplica la siguiente fórmula:

$$
P R I=R+\frac{I o-P}{Q}
$$

Dónde:

R: periodo con el último flujo negativo

Io: Inversión inicial

P: valor del último flujo mayor que la inversión

Q: valor del flujo de caja en el siguiente periodo

Tabla 6: Período de recuperación de la inversión

\begin{tabular}{crccc}
\hline Años & Flujo de Caja & Factor & $\begin{array}{c}\text { Flujo } \\
\text { Actualizado }\end{array}$ & $\begin{array}{c}\text { Flujo } \\
\text { Acumulado }\end{array}$ \\
\hline 0 & -17227.24 & 0,01 & -17727.24 & \\
1 & 199680.00 & 0.023514483 & 4695.37 & 4695.37 \\
2 & 209664.00 & 0.027302377 & 5724.33 & 10419.70 \\
3 & 220147.20 & 0.030278477 & 6665.72 & 17085.42 \\
4 & 231154.56 & 0.032553182 & 7524.82 & 24610.24 \\
5 & 242712.29 & 0.034224001 & 8306.59 & 32916.82 \\
& & & & 3 \\
\hline
\end{tabular}




\begin{tabular}{lrrr}
\hline Meses & 0.08529387 & 1.023526445 & 1 \\
& 0.023526 & 0.705793354 & 1 \\
Días & 445 & 3 Años, 1 mes, 1 día & \\
& &
\end{tabular}

Fuente: Propia (2017).

Analizando el resultado obtenido y llevándolos a meses y días como se muestra en la Tabla 6, se puede ver que el periodo de recuperación de la inversión es de 3 años, 1 mes y un día.

\section{Relación beneficio-costo}

Este indicador facilita conocer por cada dólar que se invierte en el proyecto cuanto se recupera, por lo cual se halla a partir del cociente de los ingresos en valor actualizado entre los egresos actualizados a una tasa igual a la TMAR.

$$
\text { Relación Beneficio } / \text { Costo }=\frac{\text { Ingresos Actualizados }}{\text { Egresos Actualizados }}
$$

Los ingresos actualizados aparecen en la Tabla 7 que se muestra. Aplicando la fórmula del VAN a los flujos de cada periodo y se obtiene un total de ingresos actualizados de 813488.93 USD con una tasa de incremento de un $6.56 \%$.

Tabla 7: Ingresos actualizados

\begin{tabular}{ccc}
\hline Años & Flujo de Caja & \multicolumn{1}{l}{$\begin{array}{l}\text { VAN } \\
\text { (Ingresos) }\end{array}$} \\
\hline 1 & 180480,00 & 170909,09 \\
2 & 185894,40 & 166701,10 \\
3 & 191471,23 & 162596,72 \\
4 & 197215,37 & 158593,39 \\
5 & 203131,83 & 154688,63 \\
\multicolumn{2}{c}{ Total ingresos activo. } & 813488,93 \\
\hline
\end{tabular}

Fuente: Propia. (2017).

De igual manera se aplicó la fórmula del VAN para los flujos de egresos de cada periodo obteniéndose unos egresos actualizados de 734813.73 con la misma tasa de incremento del 6.56\%. Los egresos actualizados aparecen en la Tabla 8 que se muestra.

Tabla 8: Egresos actualizados

\begin{tabular}{ccc}
\hline Años & Flujo de Caja & VAN (Egresos) \\
\hline 1 & 166180.50 & 157367.89 \\
2 & 169482.73 & 151983.91 \\
3 & 172851.01 & 146784.49 \\
4 & 176286.65 & 141763.28 \\
5 & 179791.00 & 136914.16 \\
Total egresos act. (USD) & 734813.73 \\
\hline
\end{tabular}

Fuente: Propia (2017). 
Lo cual indica que por cada dólar que se invierte en el proyecto se recupera 1.10USD. Por lo tanto, se reafirma la viabilidad del proyecto desde el punto de vista financiero.

\section{Conclusiones}

$>$ Se demostró que el proyecto es factible, teniendo un VAN de 15 189.58USD, una TIR de $30 \%$ superior al TMAR de $6.56 \%$, la inversión se recupera a los 3 años, 1 mes y un día; además de la relación beneficio costo que dio un valor de 1.10USD.

$>$ Se comprobó que los turistas que visitan el canto Pedernales se sienten atraídos por los locales polifuncionales, incluso una muestra significativa les atrae la comida costeña y se sienten identificado con un lugar que brinde múltiples opciones aperitivas y de bebida.

$>$ Con el estudio técnico se concluyó que la localización y el espacio del local es favorable para las operaciones comerciales deseadas. Lográndose determinar los recursos necesarios para poner en marcha el local polifuncional con una inversión favorable de 17 727.44USD

$>$ Se determinó que la estructura organizacional propuesta es factible para la ejecución del proyecto, con la incorporación de perfiles que se ajustan a la demanda de servicio proyectado.

$>$ Se demuestra que los locales polifuncionales permiten la diversificación de las atracciones turísticas de los clientes que visitan el cantón de Pedernales, unido al hecho que facilita la generación de empleos y la profesionalidad de los servicios

\section{Recomendaciones}

$>$ Mantener una constante capacitación en los empleados para que brinden un servicio de excelencia a los turistas que visitan el cantón, de forma tal que se logre la integralidad dentro del local.

$>$ Establecer estrategias de mejoras continuas que faciliten nuevas y eficientes formas de prestaciones y servicios ofertados en función de satisfacer a los clientes.

$>$ Incrementar la oferta de los servicios para los feriados nacionales pues el aumento de la demanda es significativo en estas fechas.

$>$ Divulgar y capacitar al resto de los propietarios de los locales conocidos como bares caipiriñas para que se incorporen a polifuncionalidad de sus espacios y servicios, con vista a homogenizar los servicios turísticos en la zona. 


\section{Referencias Bibliográficas}

Boullon. (2003). Planificación del espacio turístico. Mexico: MX. Trillas.3ed.

Doumet, Y. S. (2015). Estudio de las potencialidades turísticas para promover el desarrollo rural sostenible en el cantón pedernales.(Tesis de pregrado). Calceta: Universidad Superior Politécnica Agropecuaria de Manabí “Manuel Félix Lópe.

Ana luisa, J. (2007). Plan de Desarrollo Turismo Alternativo dentro del Cantón Pedernales, Provincia de Manabí. EC. Formato PDF. http://repositorio.ute.edu.ec/ bitstream/123456789/8509/1/33551_1.pdf

Boullon, (2003). Planificación del espacio turístico.3ed. México. MX: Trillas.

Carranza, E., y Zambrano, M. (2016). Actualización del inventario de atractivos turísticos del cantón Bolívar, provincia de Manabí. (Tesis de pregrado). ESPAM MFL. Calceta-Manabí, EC.

Doumet, Y., Shiguango R., y Vera K., (2015). Estudio de las potencialidades turísticas para promover el desarrollo rural sostenible en el cantón pedernales. (Tesis de pregrado). Universidad Superior Politécnica Agropecuaria de Manabí "Manuel Félix López”. Manabí. EC.

DRH (Desarrollo Turístico). (2011). Investigación de mercados en el campo turístico, Formato PDF. Recuperado de: http://www.hospitalitas.com/wp-content/uploads /2011/12/ Investigaci\%C3\%B3n-de-mercados-en-el-campotur\%C3\%ADstico.pdf

Dueñas, J., y Fernández, V. (2014). Modelo de gestión turístico sostenible para la planificación estratégica en el cantón Pedernales. (Tesis de pregrado). ESPAM MFL. Calceta. EC.

GAD Pedernales (Gobierno Autónomo y Descentralizado del cantón Pedernales). (2016). Información general del cantón. Pedernales. EC.

Jaramillo, A. (2016). Estudio para la creación de un restaurante polifuncional del sector turístico como alternativa dinamizadora de la economía de la zona cero del Cantón Pedernales (Tesis de pregrado). Pontifica Universidad Católica del Ecuador. Santo Domingo.

Leader. (2011). Evaluar el potencial turístico de un territorio. Formato PDF. http://ec.europa.eu/agriculture/rur/leader2/rurales/biblio/touris/metho.pdf

Malhotra, N. K. (2008). Investigación de Mercados. México. MX: Prentice Hall.

MIES. (2016). http://www.inclusion.gob.ec/ 
SENPLADES (Secretaría Nacional de Planificación y Desarrollo). (2013). Plan Nacional de Desarrollo/Plan Nacional para el Buen Vivir 2013-1017. 1ed. Quito, EC. Formato PDF. Recuperado de: http://documentos.senplades.gob.ec/Plan\%20 Nacional\%20Buen\%20Vivir\%202013-2017.pdf

MINTUR (Ministerio de Turismo del Ecuador). (2011). Turismo consciente es amar la vida. EC. Recuperado de: http://ricardomedinao.wordpress.com/2011/08/19/ turismo-consciente\%E2\%80\%A6su-definicion/

MINTUR. (2016). http://www.turismo.gob.ec/

NTE INEN 2631, (2012) http://www.normalizacion.gob.ec/wpcontent/uploads/ downloads/2013/11/alfabetico 2013.pdf

Villena, C. (2008). Marketing turístico: La segmentación. Formato HTM. Disponible en http://carlitosvillena.blogspot.com/2010/08/la-segmentacion.html (Villena, 2008) 


\section{Para citar el artículo indexado.}

Álvarez V. \& Alcívar E. (2017). Estudio para la creación de un restaurante polifuncional del sector turístico como alternativa dinamizadora de la economía en cantón Pedernales. Revista electrónica Explorador Digital 1(1), 39-60. Recuperado desde:

http://cienciadigital.org/revistacienciadigital2/index.php/exploradordigital/article/view/316/728

\section{Ciencia \\ LDigital}

El artículo que se publica es de exclusiva responsabilidad de los autores y no necesariamente reflejan el pensamiento de la Revista Explorador Digital.

El articulo queda en propiedad de la revista y, por tanto, su publicación parcial y/o total en otro medio tiene que ser autorizado por el director o editor de la Revista Explorador Digital.
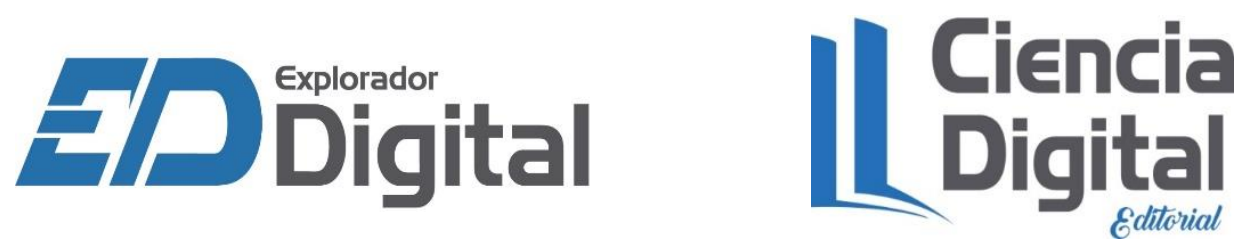